\title{
Diabetes mellitus tipo 1 infantil e as dificuldades no manejo da doença no seio familiar: Uma revisão integrativa
}

\author{
Childhood type 1 diabetes mellitus and difficulties in managing the disease in the Family sinus: An \\ integrative review \\ Diabetes mellitus tipo1 em niños y dificultades para controlar la enfermedad em seno familiar: Una \\ revisión integradora
}

Recebido: 04/06/2021 | Revisado: 14/06/2021 | Aceito: 19/06/2021 | Publicado: 30/06/2021

\author{
Sthephanine Mourão Freitas \\ ORCID: https://orcid.org/0000-0002-2832-8660 \\ Instituto de Ensino Superior de Teresina, Brasil \\ E-mail: sthephanyne_18@hotmail.com \\ Luciana Rodrigues da Silva \\ ORCID: https://orcid.org/0000-0002-3245-9567 \\ Centro Universitário do Piauí, Brasil \\ E-mail: enflucianarodriguess@ gmail.com \\ Maria Mariana Monteiro da Silva \\ ORCID: https://orcid.org/0000-0003-1654-7898 \\ Centro Universitário UNINOVAFAPI, Brasil \\ E-mail: mariamarianamds@gmail.com \\ Skarllet O'hara Pereira dos Santos \\ ORCID: https://orcid.org/0000-0003-0142-6161 \\ Centro Universitário do Piauí, Brasil \\ E-mail: skarlletohara93@gmail.com \\ Fernanda da Silva Sousa \\ ORCID: https://orcid.org/0000-0001-9571-9074 \\ Universidade Federal do Piauí, Brasil \\ E-mail: fernandassousa@live.com \\ Mercilane Alves Feitosa \\ ORCID:https://orcid.org/0000-0002-1414-9696 \\ Faculdade Estácio de Sá, Brasil \\ E-mail: mercilane_15@hotmail.com \\ Regina Márcia Soares Cavalcante \\ ORCID: https://orcid.org/0000-0002-0500-9990 \\ Universidade Federal do Piauí, Brasil \\ E-mail: reginalunna@hotmail.com
}

\begin{abstract}
Resumo
O diabetes mellitus tipol impõe a criança e seus familiares uma nova rotina, vivenciando e buscando adaptações constantes. O objetivo da pesquisa foi estudar as dificuldades enfrentadas diariamente em relação ao tratamento do DM1 infantil e os fatores que contribuem para o surgimento dessas dificuldades. Uma revisão de literatura do tipo integrativa foi realizada com levantamento de dados na Biblioteca Virtual em Saúde (BVS), Pubmed e Google Acadêmico, utilizando os seguintes descritores em inglês: "Type 1 Diabetes Mellitus", "Children`s", "Family" e em português (Diabetes Mellitus tipo 1, Infantil, Família). Foram incluídos artigos publicados nos últimos cinco anos, nacionais ou internacionais com textos completos em inglês e português. Foram selecionados inicialmente 72 artigos, dos quais 11 foram utilizados para a análise, interpretação e discussão. O estudo evidenciou que as dificuldades são diferenciadas e cada etapa depende das informações adquiridas e cuidados executados no diabetes mellitus 1 infantil e, o fator mais importante no tratamento foi o apoio familiar, com a busca contínua de informações e priorização do bemestar da criança.
\end{abstract}

Palavras-chave Diabetes Mellitus tipo 1; Infância; Família.

\section{Abstract}

Type 1 diabetes mellitus imposes a new routine on children and their families, experiencing and seeking constant adaptations. The objective of the research was to study the difficulties faced daily in relation to the treatment of childhood DM1 and the factors that contribute to the emergence of these difficulties. An integrative literature review was carried out with data collection in the Virtual Health Library (VHL), Pubmed and Academic Google, using the following descriptors in English: "Type 1 Diabetes Mellitus", "Children's", "Family" and in Portuguese (Diabetes Mellitus type 1, Infantil, Family). Articles published in the last five years, national or international, with full texts in 
English and Portuguese were included. Initially, 72 articles were selected, of which 11 were used for analysis, interpretation and discussion. The study showed that the difficulties are different and that each step depends on the information acquired and the care performed in childhood diabetes mellitus 1, and the most important factor in the treatment was family support, with the continuous search for information and prioritization of the child's well-being.

Keywords: Diabetes Mellitus type 1; Children's; Family.

\section{Resumen}

La diabetes mellitus tipo 1 impone una nueva rutina a los niños y sus familias, experimentando y buscando adaptaciones constantes. El objetivo de la investigación fue estudiar las dificultades que se enfrentan a diario en relación con el tratamiento de la DM1 infantil y los factores que contribuyen a la aparición de estas dificultades. Se realizó una revisión integradora de la literatura con recolección de datos en la Biblioteca Virtual en Salud (BVS), Pubmed y Google Académico, utilizando los siguientes descriptores en inglés: "Diabetes Mellitus tipo 1", "Niños", "Familia" y en portugués. (Diabetes Mellitus tipo 1, Infantil, Familiar). Se incluyeron artículos publicados en los últimos cinco años, nacionales o internacionales con textos completos en inglés y portugués. Inicialmente se seleccionaron 72 artículos, de los cuales 11 se utilizaron para análisis, interpretación y discusión. El estudio mostró que las dificultades son diferentes y que cada paso depende de la información adquirida y de los cuidados realizados en la diabetes mellitus infantil 1, y el factor más importante en el tratamiento fue el apoyo familiar, con la búsqueda continua de información y priorización del niño bienestar.

Palabras clave: Diabetes Mellitus tipo 1; Niño; Familia.

\section{Introdução}

Diabetes Mellitus tipo 1 (DM1) é uma doença crônica, genética, resultante da destruição das células beta pancreáticas por um processo imunológico, ou seja, pela formação de anticorpos pelo próprio organismo contra este tipo celular levando a deficiência de insulina (SBD, 2019). É mais comum na infância e adolescência e o Brasil, atualmente ocupa o terceiro lugar quanto à prevalência mundial da doença, com o quantitativo de 51.500 crianças e adolescentes com a doença, pertencentes à faixa etária de 0 a 14 anos (IDF,2019).

O diagnóstico de DM1 causa uma série de mudanças para a criança e, seus familiares devem adaptar-se a tais mudanças, para que possam seguir rigorosamente o tratamento, que consiste na administração da insulina, alimentação adequada e prática de atividade física. Apesar da doença DM1 ser menos comum que o diabetes mellitus tipo 2 (DM2), a ocorrência da doença aumenta cerca de $3 \%$ anualmente, principalmente em crianças. A monitorização da glicemia e o tratamento medicamentoso tornam-se momentos ruins, pois as agulhas usadas em ambos os procedimentos causam medo, dor, trauma e insegurança para que possam participar do momento e desejar ter o domínio para o tratamento. A educação em saúde e a riqueza de informações dos familiares, em torno da criança diagnosticada com a DM1, geram grandes avanços para o autocuidado, possibilitando a melhor forma de adequação a nova rotina, para assim manter o objetivo de equilíbrio glicêmico evitando complicações futuras (SBD, 2019).

É uma doença crônica e necessita de uma série de cuidados que gera grandes dificuldades no seio familiar, pela necessidade de mudanças no dia a dia da família e principalmente modificando por completo o estilo de vida da criança diagnosticada com DM1. Nesse contexto, o apoio profissional deve-se estender a família, para que possam construir uma base de conhecimento sobre a doença, a necessidade de mudanças do estilo de vida bem como sobre a insulinoterapia (Soares et al., 2018).

Evidencias tem demonstrado que a maior dificuldade é a insulinoterapia junto ao controle da alimentação, na busca do controle da glicemia, o que tem levado à busca da prática do autocuidado para que possam obter informações sobre a doença $\mathrm{e}$ consigam a capacitação para o exercício das atividades diárias e desenvolvimento da consciência de sua condição de diabéticos e da necessidade do autocuidado para adesão ao tratamento (Moreira et al., 2016).

O cuidado integral é de responsabilidade da família, até que a criança saiba e tenha ciência do autocuidado. O aprendizado diário é ideal para ter o conhecimento sobre a doença e limites para alcançarem a fase da autoaceitação, o apoio da equipe multiprofissional de saúde vem a somar para que assumam os cuidados até a fase do autocuidado. É importante a 
participação da família e é essencial em cada etapa para que possam avançar e assim ter o controle da doença. As dificuldades geram conflitos familiares, mas há a necessidade de que o indivíduo aceite as adaptações na rotina e a mudança em busca da qualidade de vida (Fragoso et al., 2019; Venancio et al., 2017).

O apoio familiar reforça a busca de recursos para o tratamento da doença, assim, dando espaço a saúde psicológica de todos os envolvidos realizando acompanhamento com a equipe de profissionais capacitados para orientá-los, pois dessa forma a criança abre mais espaço para a aceitação das diferentes fases e, a etapa da negação não ganha espaço quando a criança diagnosticada com DM1 se sente segura. O manejo das fases até a aceitação da doença é um processo doloroso e singular entre as famílias (Domenico \& Mendes-Castillo, 2017; Vargas et al., 2020).

É importante enfatizar que o diagnóstico da DM1 infantil causa impacto, sentimentos negativos que dominam as emoções e os tornam parte do problema, o estado emocional dos familiares quando fragilizado agrava a situação dificultando o tratamento do paciente, necessita que a família busque ajuda profissional para obter o conhecimento e o equilíbrio emocional (Ochoa et al., 2016).

Diante do exposto, o questionamento que norteou a busca de evidências na literatura cientifica especializada foi: Quais as principais dificuldades e os fatores a elas relacionadas, no manejo do diabetes mellitus tipo 1 infantil em ambiente familiar? E nessa perspectiva o objetivo deste trabalho foi verificar as dificuldades enfrentadas pela família em relação ao tratamento do DM1 infantil e os fatores que contribuem para o surgimento dessas dificuldades.

\section{Metodologia}

O estudo consiste em uma revisão de literatura do tipo integrativa, na qual utiliza-se de informações que agregam de forma compacta pesquisas relacionadas a um tema proposto (Mendes et al., 2008).

A revisão foi construída a partir de artigos que abordaram o tema diabetes infantil tipo $1 \mathrm{e}$ as dificuldades enfrentadas pela família em relação ao tratamento. A pesquisa foi conduzida no período de 25 de abril a 23 de maio de 2021 e as etapas de sua elaboração foram: 1 - Definição da questão norteadora: Quais as dificuldades enfrentadas pela família no tratamento da criança diagnosticada com a diabetes mellitus tipo 1;2 - Critérios de inclusão e exclusão: foram incluídos no estudo artigos publicados em português e inglês, nos últimos 5 anos, (2016 a 2021) e excluídos artigos que não responderam a questão norteadora do estudo; 3 - Estratégias de busca: A busca dos artigos foi realizada nas seguintes bases: Biblioteca Virtual de Saúde (BVS), Google Acadêmico e Pubmed, utilizando como descritores: "Diabetes Mellitus tipo 1", "Childrens", "Family" e correspondentes em português, adaptadas de acordo com a base de dados utilizada; 4- Seleção dos artigos: Inicialmente foram analisados os artigos pelo título e resumo, para avaliar se atendiam os critérios de inclusão e exclusão. Em caso de dúvidas, os artigos foram lidos na íntegra. Foram selecionados incialmente 72 artigos e após leitura e análise dos títulos e resumos, 11 foram selecionados e constituíram a amostra final do estudo da Figura 1. 
Figura 1. Fluxograma de identificação e seleção de artigos para revisão integrativa sobre DM1 infantil e as dificuldades enfrentadas pela família.

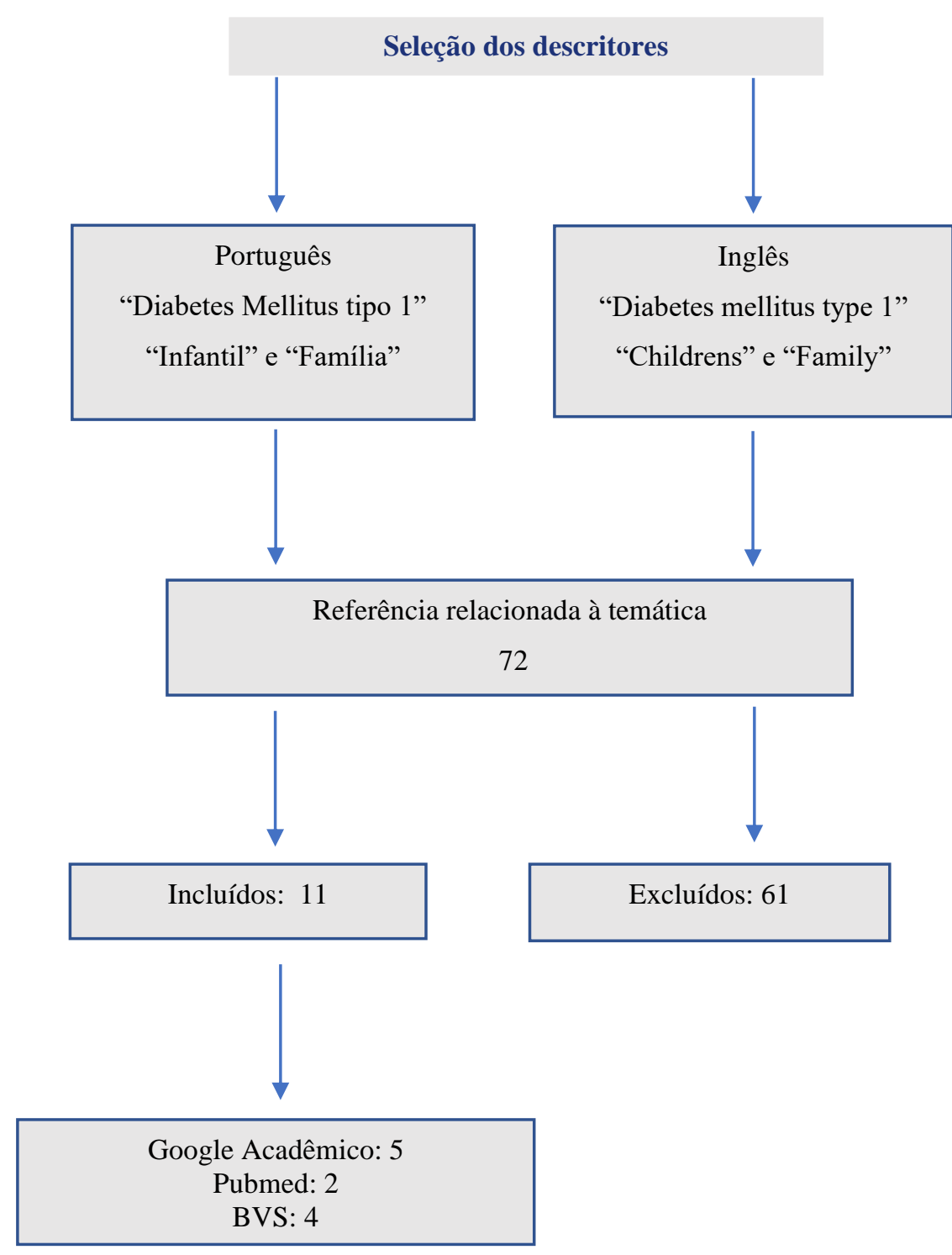

Fonte: Dados da pesquisa (2021).

\section{Resultados e Discussão}

Malaquias et al., (2016), realizou um estudo evidenciando as dificuldades, e reforçando a importância do profissional de saúde, relata que receber o diagnóstico de DM1 infantil é difícil para todos os membros da família, o resultado causa medo e sentimento de incapacidade. As famílias iniciam a busca por informações, impõe regras mais severas afim de ter resultado positivo ao tratamento, modificando o dia a dia de todos os indivíduos da família. Novas regras refletem na mudança dos hábitos alimentares e estilo de vida. 
Quadro 1: Artigos segundo autor, ano, título, objetivo, tipo de estudo e principais resultados encontrados.

\begin{tabular}{|c|c|c|c|}
\hline Autor / Título / Ano & Objetivo & Tipo de estudo & Dificuldades encontradas e Fatores contribuintes \\
\hline $\begin{array}{l}\text { Type } 1 \text { Diabetes Mellitus in } \\
\text { Pediatric Patients and Its } \\
\text { Impact on Relationships in } \\
\text { the Family Environment }\end{array}$ & $\begin{array}{l}\text { Avaliar o impacto do DM1 nas } \\
\text { relações do ambiente familiar, } \\
\text { bem como no manejo e } \\
\text { abordagem dessa doença. }\end{array}$ & $\begin{array}{l}\text { Quantitativo } \\
\text { transversal } \\
\text { descritivo }\end{array}$ & $\begin{array}{l}\text { - } \text { Mudanças no estilo de vida } \\
\text { - } \quad \text { Enfrentamento psicológico e a capacidade } \\
\text { - } \quad \text { Mo autocuidado } \\
\text { - } \quad \text { Interação da doença } \\
\text { - } \quad \text { Administração da insulina } \\
\text { - } \quad \text { Limitação as atividades diárias }\end{array}$ \\
\hline $\begin{array}{l}\text { Cuidado domiciliar à } \\
\text { criança e ao adolescente } \\
\text { com diabetes mellitus tipo } \\
1 \text { na } \\
\text { perspectiva do cuidador }\end{array}$ & $\begin{array}{l}\text { Apreender perspectivas e } \\
\text { vivências do cuidador familiar } \\
\text { sobre os cuidados prestados às } \\
\text { crianças e adolescentes com DM1 } \\
\text { no domicílio. }\end{array}$ & $\begin{array}{ll}\text { Descritivo de } & \text { do } \\
\text { abordagem } & \\
\text { qualitativa } & \end{array}$ & $\begin{array}{l}\text { - } \text { Equilíbrio psicológico } \\
\text { - } \quad \text { Falta de apoio no ambiente escolar } \\
\text { - } \quad \text { Adminiscientizaçãa em relação a doença da insulina } \\
\text { - } \quad \text { Sobrecarga física e mental do cuidador } \\
\text { - } \quad \text { Mudanças alimentares } \\
\text { - Falta de diálogo e informações sobre a } \\
\text { doença }\end{array}$ \\
\hline $\begin{array}{l}\text { Modelos explicativos das } \\
\text { famílias de crianças com } \\
\text { diabetes mellitus tipo } 1\end{array}$ & $\begin{array}{l}\text { Analisar como crianças com DM1 } \\
\text { e suas famílias explicam a } \\
\text { patologia, a partir da } \\
\text { compreensão que possuem sobre } \\
\text { os fatores relacionados à } \\
\text { descoberta do diabetes, etiologia, } \\
\text { tratamento e prognóstico da } \\
\text { doença. }\end{array}$ & Qualitativo & $\begin{array}{ll}\text { - } & \text { Autocuidado } \\
\text { - } & \text { Controle dos conflitos no seio familiar } \\
\text { - } & \text { Utilização de agulhas, tanto para o controle da } \\
\text { glicemia como para a aplicação da insulina } \\
\text { - } & \text { Alimentação adequada } \\
\text { - } & \text { Prática de exercício físico } \\
\text { - } & \text { Falta de informações relacionada a doença }\end{array}$ \\
\hline $\begin{array}{l}\text { Cuidado à criança e ao } \\
\text { adolescente com diabetes } \\
\text { mellitus tipo } 1 \\
\text { Nobre et al., } 2019\end{array}$ & $\begin{array}{l}\text { Conhecer as estratégias utilizadas } \\
\text { pela família para o cuidado à } \\
\text { criança e ao adolescente com } \\
\text { DM1. }\end{array}$ & $\begin{array}{l}\text { Qualitativo, } \\
\text { exploratório } \\
\text { descritivo }\end{array}$ & $\begin{array}{l}\text { - } \\
\text { - } \text { Aplicação da insulina } \\
\text { - } \quad \text { Descontrição alimentar } \\
\text { - } \quad \text { Rodízio dos licêmico } \\
\text { insulina }\end{array}$ \\
\hline
\end{tabular}




\begin{tabular}{|c|c|c|c|}
\hline $\begin{array}{l}\text { Crianças com diabetes } \\
\text { mellitus tipo 1: } \\
\text { Vulnerabilidade, Cuidado e } \\
\text { Acesso à Saúde } \\
\text { Wolkers et al., } 2019\end{array}$ & $\begin{array}{l}\text { Investigar a trajetória e } \\
\text { seguimento da saúde de crianças } \\
\text { com DM1 no que concerne à } \\
\text { atenção prestada pelos diferentes } \\
\text { serviços públicos de saúde e as } \\
\text { relações entre os serviços, a } \\
\text { criança e suas famílias, na } \\
\text { perspectiva de mães e cuidadores. }\end{array}$ & Qualitativo & $\begin{array}{l}\text { - } \text { Falha no atendimento profissional } \\
\text { - } \quad \text { Fragilidade no amparo a família } \\
\text { - } \quad \text { Falta de comunicação nas instituições de } \\
\text { saúde } \\
\text { - } \quad \text { Falta de qualificação profissional } \\
\text { - } \quad \begin{array}{l}\text { Insegurança nas situações de urgência e } \\
\text { emergência }\end{array}\end{array}$ \\
\hline $\begin{array}{l}\text { Criança diabética do tipo } 1 \\
\text { e o convívio } \\
\text { familiar: repercussões no } \\
\text { manejo da doença } \\
\text { Hermes et al., } 2018\end{array}$ & $\begin{array}{l}\text { Escrever a repercussão do } \\
\text { convívio familiar da criança } \\
\text { diabética no manejo da doença. }\end{array}$ & $\begin{array}{l}\text { Qualitativo } \\
\text { descritivo }\end{array}$ & $\begin{array}{l}\text { - A não aceitação da criança em relação a } \\
\text { mudança dos hábitos alimentares } \\
\text { - } \text { A não aceitação do diagnóstico pela } \\
\text { família } \\
\text { - Despreparo do genitor } \\
\text { - Comunicação efetiva entre família e os } \\
\text { profissionais de saúde }\end{array}$ \\
\hline $\begin{array}{l}\text { Autocuidado apoiado no } \\
\text { manejo da Diabetes tipo } 1 \\
\text { durante a transição da } \\
\text { infância para adolescência }\end{array}$ & $\begin{array}{l}\text { Analisar as necessidades de pré- } \\
\text { adolescentes com DM1 em } \\
\text { relação ao autocuidado apoio à } \\
\text { gestão de doenças. }\end{array}$ & $\begin{array}{l}\text { Qualitativo, } \\
\text { exploratório- } \\
\text { descritivo }\end{array}$ & $\begin{array}{l}\text { - Os desafios podem dar início ao luto } \\
\text { relacionado à doença, constituído pelos } \\
\text { estágios de negação, raiva, barganha, } \\
\text { depressão e aceitação, como destacadas nas } \\
\text { categorias sobre a negação da doença } \\
\text { - Mudanças no estilo de vida } \\
\text { - } \text { Autocuidado apoiado, autopercepção } \\
\text { distorcida de si, vergonha, medo e tristeza, } \\
\text { implicada no manejo da doença } \\
\text { - Manutenção das condições de saúde }\end{array}$ \\
\hline $\begin{array}{l}\text { Diabetes Care Provider } \\
\text { Perceptions on Family } \\
\text { Challenges of Pediatric } \\
\text { Type 1 Diabetes } \\
\text { Redondo et al., } 2017\end{array}$ & $\begin{array}{l}\text { Solicitar as perspectivas das } \\
\text { partes interessadas envolvidas no } \\
\text { cuidado de jovens com DM1 } \\
\text { sobre problemas comuns } \\
\text { relacionados à família em torno } \\
\text { do controle do diabetes e } \\
\text { estratégias sugeridas para superar } \\
\text { esses problemas. }\end{array}$ & Qualitativo & $\begin{array}{ll}\text { - } & \text { Independência para o autocuidado } \\
\text { - } & \text { Negação } \\
\text { - } & \text { Conflito familiar } \\
\text { - } & \text { Quebra da comunicação efetiva }\end{array}$ \\
\hline $\begin{array}{l}\text { Vivências de mães de } \\
\text { crianças diabéticas } \\
\text { Cruz et al., } 2017\end{array}$ & $\begin{array}{l}\text { Compreender a vivência de mães } \\
\text { de crianças com DM1. }\end{array}$ & Qualitativo & $\begin{array}{l}\text { - Convivência conflituosa } \\
\text { - Dificuldade no manejo dos materiais para a } \\
\text { verificação da glicemia e administração da } \\
\text { insulina } \\
\text { - Privações e controle da alimentação } \\
\text { - Mudanças e informações sobre o estilo de } \\
\text { vida }\end{array}$ \\
\hline
\end{tabular}




\begin{tabular}{|l|l|l|ll|}
\hline $\begin{array}{l}\text { As demandas de cuidado } \\
\text { das crianças com Diabetes } \\
\text { Mellitus tipo 1 }\end{array}$ & $\begin{array}{l}\text { Conhecer a experiência de } \\
\text { famílias no cuidado às crianças } \\
\text { com DM1. }\end{array}$ & $\begin{array}{l}\text { Descritivo e } \\
\text { exploratório com } \\
\text { abordagem } \\
\text { qualitativa. }\end{array}$ & $\begin{array}{l}\text { Restrição alimentar } \\
\text { Dificuldade financeira }\end{array}$ \\
Okido et al, 2017 & $\begin{array}{l}\text { Dificuldade na aplicação da insulina } \\
\text { - Ambiente escolar despreparado para }\end{array}$ \\
\hline $\begin{array}{l}\text { Atenção primária à criança } \\
\text { com diabetes mellitus tipo } \\
\text { 1: perspectiva de } \\
\text { cuidadores }\end{array}$ & $\begin{array}{l}\text { Avaliar e comparar a qualidade } \\
\text { da atenção primaria ofertada as } \\
\text { crianças com DM1 entre os tipos } \\
\text { de serviços públicos de atenção à } \\
\text { saúde na experiencia dos seus } \\
\text { principais cuidadores. }\end{array}$ & Transversal & $\begin{array}{l}\text { Fragilidade no vínculo entre profissional e } \\
\text { família }\end{array}$ \\
Wolkers et al., 2017 & $\begin{array}{l}\text { Agendamentos de consultas com tempo de } \\
\text { espera prolongado }\end{array}$ \\
\hline
\end{tabular}

Fonte: Dados da pesquisa.

O estudo realizado por Okido et al., (2017), sobre as demandas de cuidado das crianças com Diabetes Mellitus tipo 1, revelaram as dificuldades enfrentadas pela família ao cuidarem da criança diagnosticada com DM 1. Observou-se que desde o diagnóstico, a família deve aliar-se com dedicação, cuidado e apoio, para reforçar o controle da doença crônica infantil. Além disso, verificou-se que as dificuldades não estão relacionadas somente ao tratamento da doença no seio familiar, mas também financeira. Foram analisadas as dificuldades diárias enfrentadas pelas famílias e abordadas a busca por alimentos, qual a melhor opção a oferecer, regrando e se adequando a alimentação de qualidade, modificando os hábitos alimentares de todos. O cuidado diário incluiu a monitorização da glicemia, para determinar o nível de controle glicêmico do paciente, assim identificando e prevenindo a hipoglicemia e hiperglicemia.

Cruz et al., (2017), realizaram um estudo com mães de crianças diabéticas e reforçou a importância da integração da equipe profissional do cuidado integral a família. As mães apresentaram sinais de cansaço e estresse devido ao cuidado e atenção integral dada a criança, levando em si um nível alto de responsabilidade e o medo da perda. A equipe esclareceu e tirou todas as dúvidas da família, expressando a importância do diálogo com todos os membros, incluindo a criança diabética para que juntos pudessem adaptar-se a nova rotina. $\mathrm{O}$ estudo esclareceu os significados da mudança relatando a rotina das mães sobre o diálogo do dia a dia.

Souza et al., (2020), em pesquisa com 11 cuidadores residentes em município no Noroeste do Paraná, verificaram que as dificuldades de mães e pais e se estendeu ao ambiente escolar e que não tinham preparo para dar suporte ao aluno diabético, impossibilitando que a criança frequentasse a escola. Foi observado a falta de conhecimento dos profissionais sobre a doença, manuseio, e aplicação da insulina. Observou-se também que a alimentação não era apropriada, levando uma preocupação adicional a essas famílias. Os esforços demandados por esse cuidado conduziram a mãe para a desistência do seu trabalho, renunciando a sua renda mensal para assim cuidar integral do filho diabético.

Sobre o cuidado a criança e ao adolescente, estudo de Nobre et al., (2019), analisou a importância do acompanhamento profissional junto a família da criança diagnosticada com DM1, que fortificou a comunicação com outras famílias para trocas de experiências diárias, expondo assim, suas principais dificuldades e novas necessidades. Foi enfatizado que a criança tem que aprender a lidar com a situação junto aos membros da família, que é a principal fonte de apoio para lidar com a fase de negação, pois a família e o apoio profissional irão proporcionar o conhecimento e informações sobre a doença, segurança para 
a aplicação da insulina como também estimulará o cuidado e atenção no manuseio e armazenamento de todo o material para o controle da doença.

De acordo com Hermes et al., (2018), o responsável nem sempre tem ações positivas e assertivas referentes a doença para que possa ter esse controle. Ao analisar o estudo de caso em seguimento ambulatorial, por meio de grupo focal, foi identificado que muitos não estão preparados para levar adiante o tratamento da DM1, por causa de condições inapropriadas do genitor, e dessa forma um outro familiar assume a guarda, entretanto, não manifesta comprometimento integral com a causa e consequentemente não busca o controle da doença e meios para que possa controlar a criança, doença e a situação a qual está unida. Nessa perspectiva foi mostrado que há a necessidade de fortalecimento de vinculo e abertura para a busca de ajuda e conhecimento, estabelecimento equilíbrio para a obtenção de resultados satisfatórios em relação ao controle da doença.

Evidências demonstraram que na infância e adolescência o cuidado pessoal de portadores de DM1, gera muitas dúvidas, medos e constrangimentos em relação à doença. A fase de negação é visível e assume a vez, pois os mesmos não buscam falar sobre a doença, devendo a família ter o cuidado e somar as informações que necessitam, diariamente, em relação ao manejo adequado da referida doença, apoiando em cada etapa a ser estabelecida para que possam se fortalecer e ter esse cuidado pessoal dentro e fora de casa. Foi identificado que adolescentes que passaram pela fase de negação buscam ouvir novas experiências para que possa fortalecer o autocuidado (Collet et al., 2018).

Pesquisa conduzida por Wolkers et al., (2019), revelou que as dificuldades enfrentadas por pais ou responsáveis de crianças com DM1, o despreparo dos profissionais em relação ao atendimento emergencial à criança diabética e comunicação a família, assim como o medo e a desconfiança dos pais a frente de tais situações. Em decorrência desse aumento de doenças crônicas no mundo, torna-se cada vez mais relevante a existência de orientações adequadas, redes de apoio a família e treinamento a esses profissionais. As práticas de educação em saúde devem ser expostas a essas famílias, para promover a melhoria e controle da patologia. Entretanto essa visão negativa leva as famílias a não buscar essa atenção especializada à saúde e somente o atendimento eletivo com o profissional que desperte confiança.

Analisando o estudo de Madrigal et al., (2020), foram observados comportamentos e sentimentos diferentes nas distintas faixas etárias. Para os menores de cinco anos, o medo e o manuseio na aplicação da insulina tornavam mais difícil o autocuidado. Quanto maior a faixa etária mais informação e maturidade é adquirida, mas esse processo de aprendizagem causa momentos de constrangimentos assim que surgem dúvidas referente a doença. Por esse motivo deve-se tornar o compartilhamento livre e que deixe a criança relatar seus sentimentos em relação à doença e seu manuseio. Mesmo limitando em certas atividades ou restringindo a alguns alimentos, algumas crianças não sentiram dificuldades de relacionar-se com outras pessoas ou ambiente e adequaram-se com mais facilidade a nova rotina.

O comportamento positivo da família foi analisado no estudo de Redondo et al., (2017), contribuiu para bons resultados ao tratamento e diminuição dos desentendimentos. Entender as limitações é ideal para ajudar os pacientes nessa batalha diária que é o enfrentamento da doença. A recomendação é a comunicação efetiva, acompanhamento psicológico e apoio total para que seja incentivado o autocuidado.

O acesso a saúde, segundo estudo de Wolkers et al., (2017), necessita ser modificado diminuindo ou excluindo as limitações para que possa atender e suprir as necessidades da criança diabética. $\mathrm{O}$ acesso a marcação de consultas ou demandas urgentes necessita de facilidade, para prolongar a continuidade a assistência e fornecer segurança ao paciente, e os profissionais devem atuar com responsabilidade e atenção a essas crianças.

O diagnóstico do DM1 altera a qualidade de vida da família ou responsável pela criança, tornando esse período desafiador e levando modificações no seio familiar, gerando conflitos e dificuldades proporcionados por uma série de fatores. O desconhecido gera a negação, necessitando de informações que enriqueçam e tomem o controle para seguir o tratamento 
levando a mudança de hábitos alimentares e o estilo de vida. As famílias se adaptaram a nova rotina, cada uma no seu tempo, com suas diferenças, dando espaço ao equilíbrio emocional para o alcance de seu objetivo (Dantas et al., 2020).

\section{Considerações Finais}

A pesquisa evidenciou dificuldades existentes no seio familiar que desestabilizava a rotina de todos os seus membros, modificando significativamente a rotina da criança diagnosticada com diabetes mellitus tipo $1 \mathrm{e}$ dos seus familiares. A falta de informações relacionadas à doença pode gerar medo e desencadear problemas psicológicos aos cuidadores e para criança. E nesse contexto é fundamental analisar a composição do núcleo familiar, o papel desempenhado por cada relacionado ao tratamento, considerando que o suporte familiar é importante para a construção da autonomia da criança e aceitação da doença.

$\mathrm{O}$ estudo mostrou ainda que as dificuldades surgem a partir do diagnóstico da doença, trazendo modificações e incertezas no seio familiar. As adaptações à nova rotina exigem disciplina rigorosa e, dessa forma, os pais necessitam de orientação e apoio para lidar, satisfatoriamente, com as imposições desta nova fase, pois a criança necessita ser orientada e acompanhada pela família e a equipe multiprofissional a fim de poder manter o índice glicêmico em valores mais próximos possíveis dos normais.

É oportuno enfatizar que a participação da equipe multiprofissional gera resultados satisfatórios e, a assistência integral deve ser estendida a todos os membros da família, levando o conhecimento para diminuir as dificuldades no ambiente familiar. Os profissionais são responsáveis pelo planejamento e implementação de estratégias que facilitem o cuidado, capacitando a família e encorajando a criança nessa fase. E assim espera-se que todos os membros do núcleo familiar da criança a escutem e apoiem, como também observem atentamente seu comportamento e busque estratégias que melhorem sua qualidade de vida, tornando menos assustador o controle da glicemia com estímulos constantes ao autocuidado, e dessa forma, tornar mais leve e mais próximo da normalidade, o dia a dia dessa criança. Assim, a grande contribuição deste trabalho foi a realização do levantamento de importantes informações que podem subsidiar a orientação dos profissionais e familiares que lidam com crianças acometidas com DM1, como também por estimular a realização de mais estudos com maior abrangência sobre o referido tema.

\section{Referências}

Collet, N., Batista, A. F. de M. B., da Nóbrega, V. M., Souza, M. H. do N., \& Fernandes, L. T. B. (2018). Self-care support for the management of type 1 diabetes during the transition from childhood to adolescence. Revista Da Escola de Enfermagem, 52. https://doi.org/10.1590/S1980-220X2017038503376

Cruz, D. S. M. da, Collet, N., Maria Costa de Andrade, E., Medeiros da Nóbrega, V., Miriam Lima da Nóbrega, M., \& Anna, E. (2017). Vivências de mães de crianças diabéticas a Mothers of experiences in diabetic child Experiencias de madres de niños diabéticos PESQUISA | RESEARCH. Escola Anna Nery, 21(1), 20170002. https://doi.org/10.5935/1414-8145.20170002

Dantas, I. R. de O., Neris, R. R., Zago, M. M. F., Santos, M. A. Dos, \& Nascimento, L. C. (2020). Explanatory models of families of children with type 1 diabetes mellitus. Revista Brasileira de Enfermagem, 73 4, e20180975. https://doi.org/10.1590/0034-7167-2018-0975

Domenico, C. T. De, \& Mendes-Castillo, A. M. C. (2017). Apoio social da criança com diabetes tipo 1 e sua família. Revista de Enfermagem UFPE on Line, 11(12), 5020. https://doi.org/10.5205/1981-8963-v11i12a23166p5020-5027-2017

Fragoso, L. V. C., Cunha, M. da C. dos S. O., Fragoso, E. B., \& Araújo, M. F. M. de. (2019). Self-Care Among Type 1 Diabetes Mellitus Bearing People: Adolescents' Experience. Rev. Pesqui. Cuid. e Fundam., 11(2), 289-296. https://doi.org/10.9789/2175-531.2019.v11i2.289-296

Hermes, T. S. V., Viera, C. S., Rodrigues, R. M., Toso, B. R. G. de O., \& Fonseca, L. M. M. (2018). Criança diabética do tipo 1 e o convívio familiar: repercussões no manejo da doença. Saúde Em Debate, 42(119), 927-939. https://doi.org/10.1590/0103-1104201811911

IDF. International Diabetes Federation. Diabetes Atlas, 9 th. Brussels, Belgium: 2019. Disponível em: http://www.diabetesatlas.org. Acesso em: 23 de maio de 2021.

Madrigal, M. A., López, M., Sánchez, A., Cao, M. J., Castro, M. J., \& María Jiménez, J. (2020). Type 1 Diabetes Mellitus in Pediatric Patients and Its Impact on Relationships in the Family Environment. https://doi.org/10.2147/DMSO.S281949

Malaquias, T. D. S. M., Marques, C. D. C., Faria, A. C. de P., Pupulim, J. S. L., Marcon, S. S., \& Higarashi, I. H. (2016). A criança e o adolescente com diabetes mellitus tipo 1: desdobrar do cuidado familiar. Cogitare Enfermagem, 21(1). https://doi.org/10.5380/ce.v21i4.42010 
Mendes, karina D. S., Silveira, R. C. D. C. P., \& Galvão, C. M. (2008). Out-Dez (Vol. 17, Issue 4).

Moreira, T. R., Torquato, S., Bandeira, A., Lopes, S. C., Linhares De Carvalho, S., Diana Da, F., Negreiros, S., Da, C., \& Neves, S. (2016). Dificuldades de crianças e adolescentes com Diabetes Mellitus tipo 1 acerca da doença Difficulties concerning Diabetes Mellitus Type 1 in children and adolescents. Rev Rene, 17(5), 651-658. https://doi.org/10.15253/2175-6783.2016000500010

Nobre, C. M. G., Rodrigues Costa, A., Minasi, A. S., Medeiros Possani, S., Soares Mota, M., \& Gomes, G. C. (2019). Care for Children and Adolescents With Diabetes Mellitus Type 1 Cuidado À Criança E Ao Adolescente Com Diabetes Mellitus Tipo 1 Cuidado Al Niño Y Al Adolescente Con Diabetes Mellitus Tipo 1. 13(1), 111-118. https://doi.org/10.5205/1981-8963-v13i01a238622p111-117-2019

Ochoa, M., Cardoso, M., \& Reyes, V. (2016). Emociones de la familia ante el diagnóstico de diabetes mellitus tipo 1 en el infante. Enfermería Universitaria, 13(1), 40-46. https://doi.org/10.1016/j.reu.2016.01.006

Okido, A. C. C., Almeida, A. de, Vieira, M. M., Neves, E. T., Mello, D. F. de, \& Lima, R. A. G. (2017). Care demands of children with type 1 Diabetes Mellitus. Escola Anna Nery - Revista de Enfermagem, 21(2), 20170034. https://doi.org/10.5935/1414-8145.20170034

Redondo, M. J., Callender, C. S., Gonynor, C., Cantu, D., Cullen, K. W., Anderson, B., \& Thompson, D. (2017). Diabetes care provider perceptions on family challenges of pediatric type 1 diabetes. Diabetes Research and Clinical Practice, 129, 203-205. https://doi.org/10.1016/j.diabres.2017.05.006

SBD. Sociedade Brasileira de Diabetes. Diretrizes da Sociedade Brasileira de Diabetes 2019-2020. São Paulo: Editora Clannad, 2019. https://www.diabetes.org.br/profissionais/images/DIRETRIZES-COMPLETA-2019- 2020.pdf.

Soares, N. S., Rocha, T. K. da, Carvalho, G. P. de, Viegas, K., Geremia, C., \& Canabarro, S. T. (2018). Conhecimento dos familiares de crianças com diabetes mellitus 1 atendidas em um centro de referência. Research, Society and Development, 7(7), e677294. https://doi.org/10.17648/rsd-v7i7.294

Souza, R. R., Marquete, V. F., Vieira, V. C. de L., Fischer, M. J. B., Spigolon, D. N., \& Marcon, S. S. (2020). Home care for child and adolescent with type 1 diabetes mellitus from the care giver's perspective. Revista Enfermagem, 28(0), 1-6. https://doi.org/10.12957/reuerj.2020.46013

Vargas, D. M., Barbaresco, A. C., Steiner, O., \& Silva, C. R. L. D. da. (2020). Um Olhar Psicanalítico Sobre Crianças e Adolescentes com Diabetes Mellitus Tipo 1 e seus Familiares. Revista Psicologia e Saúde, 12(1), 87-100. https://doi.org/10.20435/pssa.v12i1.858

Venancio, J. M. P., Banca, R. O. La, \& Ribeiro, C. A. (2017). Benefits of participation in a summer camp to self-care for children and adolescents with diabetes: the perception of mothers. Escola Anna Nery - Revista de Enfermagem, 21(1), 20170004. https://doi.org/10.5935/1414-8145.20170004

Wolkers, P. C. B., Macedo, J. C. B., Rodrigues, C. M., De Carvalho Furtado, M. C., \& De Mello, D. F. (2017). Atenção primária à criança com diabetes mellitus tipo 1: Perspectiva de cuidadores. ACTA Paulista de Enfermagem, 30(5), 451-457. https://doi.org/10.1590/1982-0194201700066

Wolkers, P. C. B., Pina, J. C., Wernet, M., Furtado, M. C. de C., \& de Mello, D. F. (2019). Children with diabetes mellitus type 1: Vulnerability, care and access to health. Texto e Contexto Enfermagem, 28. https://doi.org/10.1590/1980-265x-tce-2016-0566 